\title{
REGISTRATION OF MULTIPLE STAINED HISTOLOGICAL SECTIONS
}

\author{
Jiř́ Borovec ${ }^{1}$, Jan Kybic ${ }^{1}$, Michal Bušta ${ }^{1}$, Carlos Ortiz-de-Solórzano ${ }^{2}$, Arrate Muñoz-Barrutia ${ }^{2}$ \\ ${ }^{1}$ Czech Technical University in Prague, Czech Republic \\ ${ }^{2}$ Center for Applied Medical Research, University of Navarra, Pamplona, Spain
}

\begin{abstract}
The analysis of protein-level multigene expression signature maps computed from the fusion of differently stained immunohistochemistry images is an emerging tool in cancer management. Creating these maps requires registering sets of histological images, a challenging task due to their large size, the non-linear distortions existing between consecutive sections and to the fact that the images correspond to different histological stains and thus, may have very different appearance. In this manuscript, we present a novel segmentation-based registration algorithm that exploits a multi-class pyramid and optimizes a fuzzy class assignment specially designed for this task. Compared to a standard nonrigid registration, the proposed method achieves an improved matching on both synthetic as well as real histological images of cancer lesions.
\end{abstract}

Index Terms - Non-rigid registration, superpixel, multiclass matching, light microscopy images, lung cancer

\section{INTRODUCTION}

Molecular phenotyping of cancer lesions based on multi-gene expression signature maps is increasingly being used for diagnosis, prognosis and personalized therapy planning. In experimental settings, this is done by multivariate molecular profiling, based on gene expression arrays. However, due to cost and time constraints, immunohistochemistry (IHC) is the method of choice in clinical settings. Metzger et al. [1] recently developed a platform that computes multiple-gene expression signature maps from consecutive IHC sections digitized using a commercial whole-slide imaging system. The critical step in this process is the correct assignment of spatial correspondence between differently stained sections. In their platform, the IHC images were aligned to a reference Hematoxylin and Eosin (H\&E) image through a two-step process involving coarse manual alignment followed by an automatic fine rigid registration using TurboReg ${ }^{1}$. Nevertheless, histological images often suffer from non-linear

The project was partially supported by Czech Science Foundation under project P202/11/0111, Grant Agency of the Czech Technical University in Prague, grant No.SGS12/190/OHK3/3T/13, the UTE Project CIMA, MICINN DPI2009-14115-C03-03 and MINECO DPI2012-38090-C03-02.

${ }^{1}$ http://bigwww.epfl.ch/thevenaz/turboreg/ distortions (i.e., missing sections, tissue folding, stretching and tearing) that cannot be corrected for using a rigid transformation. An elastic registration of histological sections was introduced in [2]. Furthermore, other issues complicate the registration process, such as the very different appearance and local architecture of the sections and the considerable size of the images. Aware of that, we previously implemented a non-rigid intensity-based registration method initialized by the matching of small salient anatomical features based on ITK, Elastix ${ }^{2}$ and OpenCV. Although it results in better registration quality than methods based on rigid registration, this method is not able to fully capture local deformations.

In this paper, we present a novel non-rigid segmentationbased registration algorithm based on a multi-class segmentation pyramid. We demonstrate that this method improves the results of the reference method on both synthetic and real histological images of cancer lesions.

The structure of the paper is as follows. Section 2 describes the real and synthetic images. In Section 3, both the intensity-based and the segmentation-based registration methods are described. Section 4 presents the evaluation results. The paper ends with the concluding remarks in Section 5.

\section{MATERIAL}

In this section, the sample preparation and image acquisition is described, together with the generation of the synthetic image pairs.

\subsection{Real images}

We used lung tissue blocks from a long-term urethaneinduced lung cancer mouse model. Unstained adjacent $3 \mu \mathrm{m}$ formalin-fixed paraffin-embedded sections were cut from the blocks and stained with Hematoxylin and Eosin (H\&E) or by immunohistochemistry with a specific antibody for CD31, proSPC, CC10 or Ki67 (see Figure 1). Images of two lesions were acquired with a Zeiss Axio Imager M1 microscope (Carl Zeiss, Jena, Germany) equipped with a $d r y$ Plan Apochromat objective (numerical aperture $N A=0.95$, magnification $40 \times$, pixel size $0.174 \mu \mathrm{m} /$ pixel, tile size $2080 \times 1540$ pixels). Lesion 1 (lesion 2$)$ occupies $9 \times 10(9 \times 9)$ tiles. The

\footnotetext{
2http://elastix.isi.uu.nl/
} 


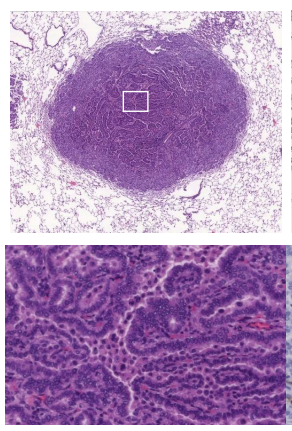

(a)
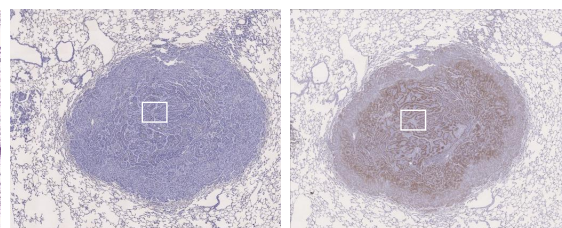

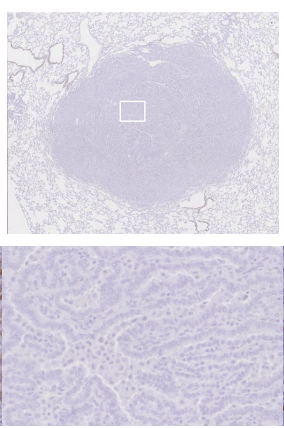

(d)

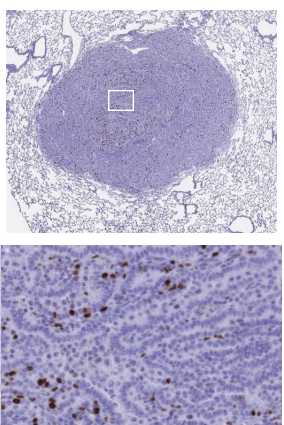

(e)

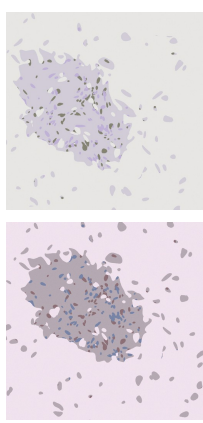

(f)

Fig. 1. (Top row) Sample mosaic $(18720 \times 15400$ pixels) showing a typical lung cancer lesion. (Bottom row) Zoomed area showing the different appearance and local structure of the same area in consecutive slices. (a) H\&E staining: nuclei are purple, cytoplasm is pink and vessels are red; Immunochemistry stains: Negative nuclei and cytoplasm are colored in dark and light blue, respectively. Positive content (nuclei/cytoplasm) is shown in dark brown. (b) CD31: stains the endothelial cells that form blood vessels; (c) proSPC: type 2 pneumocytes forming the alveolar-capillary barrier; (d) CC10: clara cells found in the small airways (bronchioles); (e) Ki67: nuclei of growing dividing cells. (f) Example of a synthetic reference and moving image pair.

Table 1. Generation of the synthetic images. The last column refers to the maximal displacement of each control point in a B-spline grid (of size $50 \times 50$ pixels). Percentages are given with respect to the image size $(1600 \times 1600$ pixels $)$.

\begin{tabular}{clccc}
\hline Dataset & Transform. & Rot. & Translat. & Disp. \\
\hline 1 & Rigid & $\pm 10^{\circ}$ & $\pm 5 \%$ & - \\
2 & Non-rigid & $\pm 10^{\circ}$ & $\pm 5 \%$ & $\pm 5 \%$ \\
3 & Rigid & $\pm 20^{\circ}$ & $\pm 10 \%$ & - \\
4 & Non-rigid & $\pm 20^{\circ}$ & $\pm 10 \%$ & $\pm 10 \%$ \\
\hline
\end{tabular}

mosaic images of the whole lesions were composed using Fiji Stitcher tool ${ }^{3}$.

\subsection{Synthetic images}

We generated four synthetic image datasets (each has 100 pairs) mimicking consecutive histological sections. Each image pair is composed of a moving $M$ and a reference image $R$ (see Figure 1 (f)). To create each pair, first a segmentation of a moving segmentation of size $1600 \times 1600$ pixels was generated containing four classes of symbolic structures (background, vessels and positive/negative content (either nuclei or cytoplasm)). Next, the reference segmentation was obtained by distorting the moving segmentation with one of the four random transformations shown on Table 1. Each image was randomly assigned to a different stain and the segmentation classes colored accordingly. To get a textured pattern, $5 \%$ white noise was added to the images.

\section{METHODOLOGY}

In this section, we describe the main functional blocks (Figure 2) of the two compared pairwise registration algorithms.

\footnotetext{
${ }^{3}$ http://fiji.sc/wiki/index.php/stitching_2D/3D
}

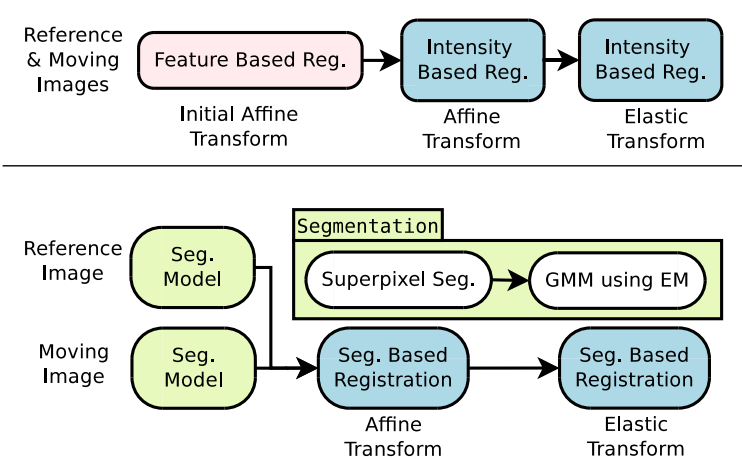

Fig. 2. Flowchart of the compared algorithms: (Top) Reference method. (Bottom) Segmentation-based registration.

\subsection{Reference method}

Features are detected and extracted by the Speeded Up Robust Feature (SURF) method [3]. Then, on the feature pairs, Random Sample Consensus (RANSAC) is used to eliminate outliers. An initial, coarse affine transform is estimated by least-squares error minimization on the feature set positions.

An affine transformation is estimated coarsely by least squares fitting of the feature point positions. The affine estimate is refined and a final elastic B-spline transformation is found using ITK/Elastix multi-resolution registration implementation with Limited-memory Broyden-FletcherGoldfard-Shanno (LBFGS) optimizer and mutual information metric using a random coordinate sampling in every iteration.

\subsection{Segmentation-based registration}

Here, we propose a multi-scale non-rigid segmentation-based registration algorithm maximizing a fuzzy class assignment. Segmentation pyramids are independently constructed for the two images to be registered (reference and moving image). 


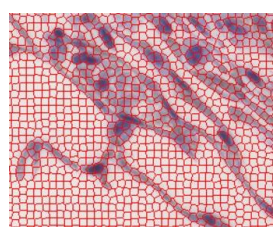

(a)

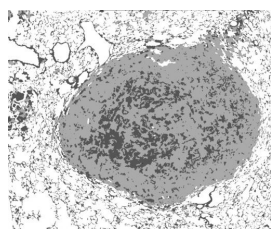

(b)

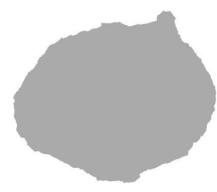

(c)
Fig. 3. For a CD31 stained image sample (see Figure 1(b)): (a) Detail of our superpixels segmentation; Illustration of the multi-class pyramid: (b) Intermediate level. (c) Coarsest level.

Each level of the pyramid contains a multi-class segmentation capturing image structures at a given scale. At each level of the pyramid, the segmentation of the reference and moving images needs to represent the same type of structures as the registration similarity metric relies on the correct class assignments. This is guaranteed through a combined class merging and matching mechanism. As in a conventional multiresolution registration, the transformation is estimated at the coarsest level of the pyramid and then, sequentially propagated to the next levels to refine the solution.

Notation. Let $I: \Omega \rightarrow \mathbb{R}^{3}$ be a continuous RGB image defined over a 2-dimensional discrete image domain $\Omega=$ $\left[1, \ldots, n_{x}\right] \times\left[1, \ldots, n_{y}\right]$ where $n_{x}$ and $n_{y}$ are the image size in the $x$ - and $y$-dimensions, respectively. We will index it with a running index $i$ as ordering is not relevant in our case. The labeling function $Y: \Omega \rightarrow L$ assigns to each pixel in $\Omega$ a label from a set of labels $L$. The binary segmentation $\mathbb{I}_{c}(Y)$ for a label $c \in L$ is defined as

$$
\mathbb{I}_{c}(Y)(i)= \begin{cases}1 & \text { if } Y(i)=c \\ 0 & \text { elsewhere }\end{cases}
$$

Namely, $\mathbb{I}_{c}(Y): \Omega \rightarrow\{0,1\}$. The transformation function $g$ maps the moving segmented image $Y^{M}$ to the reference one $Y^{R}$ such as $g: \Omega^{M} \rightarrow \Omega^{R}$.

Segmentation model. Here, we present the segmentation pipeline summarized in Figure 2 (bottom). First, superpixels are computed using the Simple Linear Iterative Clustering (SLIC) algorithm [4] (see Figure 3 (a)). Each superpixel is described by the mean intensity of the pixels that belong to it. This superpixel-based simplified representation of the image is fed to an Expectation-Maximization (EM) algorithm that estimates a four class Gaussian Mixture Model (GMM). From the probability model, a compact 6-level multi-class segmentation pyramid (see Figure 3 (b) and (c)) is computed using Graph Cuts segmentation [5] with smoothness coefficients logarithmically distributed in the range $[1,100000]$.

Label merging and matching. To properly compute the registration similarity metric, the number of labels and their order for both the reference and moving images need to be the same. Label merging equates the number of labels. Before presenting the merging criterion, we need to define the association metric $S_{c, d}$ for labels $c \in L$ and $d \in L$

$$
\begin{aligned}
S_{c, d}=\frac{|(C \cup D) \backslash(C \cap D)|}{|C \cup D|} \cdot\left(1-\frac{|C|}{|D|}\right) & \\
& \text { with }|D| \geq|C|
\end{aligned}
$$

where $C=\mathbb{I}_{c}\left(Y^{A}\right), D=\mathbb{I}_{d}\left(Y^{B}\right), Y^{A}$ and $Y^{B}$ represent the segmented image pair, $|\cdot|$ the non-zero pixel count, $\cup$ represents the union operator, $\cap$ the intersection and $\backslash$ the difference. We have $S_{c, d} \in[0,1]$ with a value close to zero indicating a high overlap and a similar area extension of both classes.

Let $D$ be the binary segmentation $D=\mathbb{I}_{d_{1}}\left(Y^{B}\right) \cup$ $\mathbb{I}_{d_{2}}\left(Y^{B}\right)$. The labels $d_{1} \in L$ and $d_{2} \in L$ in $Y^{B}$ are merged iff $\min _{c} S_{c, d} \ll \eta \cdot \min _{c} \min \left(S_{c, d_{1}}, S_{c, d_{2}}\right)$, with $c \in L$ and $\eta=5$.

Class matching is also based on the computation of the association metric $S$ and the best matching is found with the Hungarian algorithm [6].

Registration. An affine transformation is computed and further refined by a non-rigid B-spline transformation. To avoid discontinuities in the optimization function, we work with the fuzzy segmentations obtained by applying a Gaussian filter $H$ to the binary segmentation $Y: \mathbb{I}_{c}(Y)^{*}=$ $H\left(\mathbb{I}_{c}(Y)\right)$. The similarity metric is defined as

$$
J=\frac{\sum_{c \in L} \sum_{i \in \Omega} \mathbb{I}_{c}^{*}\left(Y^{R}\right)(i) \cdot\left(g \circ \mathbb{I}_{c}^{*}\left(Y^{M}\right)(i)\right)}{\sum_{c \in L} \sum_{i \in \Omega}\left(g \circ \mathbb{I}_{c}^{*}\left(Y^{M}\right)(i)\right)}
$$

Finally, the transformation $\hat{g}$ is estimated as the one maximizing the fuzzy class assignment as measured by $J$ (i.e., $\hat{g}=\arg \max _{g} J$ ) using a gradient descent optimization. We stop iterating when the increment of $J$ is smaller than a given threshold $\left(\epsilon=10^{-4}\right)$.

\section{EXPERIMENTAL RESULTS}

\subsection{Synthetic images}

The registration on synthetic images (see Figure 4 (a)-(c)) was evaluated using the mean Euclidean distance between the positions of the contour points in the reference and the transformed moving image. The results are shown in Table 2. We observed that the proposed method was more robust to strong deformations than the reference method.

\subsection{Real images}

For the evaluation of the registration results on real images (see Figure 4 (d)-(f)), we used manually placed landmarks. 


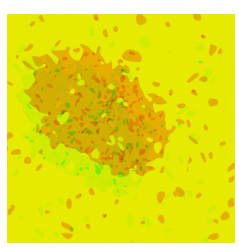

(a)

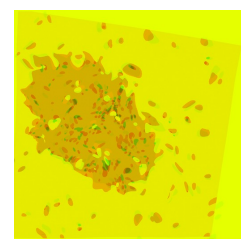

(b)

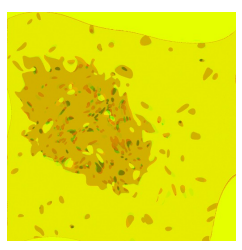

(c)

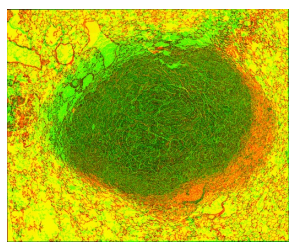

(d)

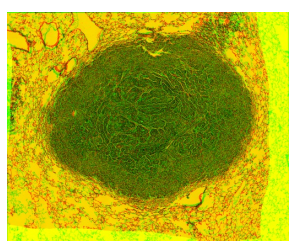

(e)

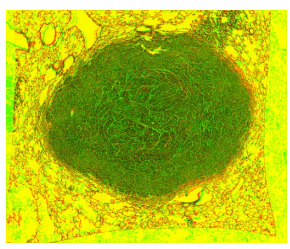

(f)

Fig. 4. Visual estimation of the registration accuracy. The reference (moving) image is shown in green (red). (Left) Synthetic images. (Right) Real images: (a) (d) Superposition of the input image pair. (b) (e) Reference registration output. (c) (f) Segmentation-based registration output.

Table 2. Registration errors for the synthetic images measured as the mean Euclidean distance between the positions of the contour points.

\begin{tabular}{llcccc}
\hline \multirow{2}{*}{ Methods } & Error & \multicolumn{4}{c}{ Dataset } \\
& (pixels) & 1 & 2 & 3 & 4 \\
\hline \multirow{2}{*}{ Ref-Reg. } & Mean & 0.77 & 10.74 & 6.33 & 53.93 \\
& Std & 0.38 & 5.87 & 22.09 & 49.82 \\
\hline \multirow{2}{*}{ Segm-Reg. } & Mean & 0.18 & 9.01 & 1.52 & 22.11 \\
& Std & 0.09 & 7.69 & 12.09 & 16.25 \\
\hline
\end{tabular}

Table 3. Registration errors for the real images measured as the mean Euclidean distance between the positions of the manually placed landmarks.

\begin{tabular}{llcc}
\hline \multirow{2}{*}{ Methods } & Error & \multicolumn{2}{c}{ Lesion } \\
& (microns) & 1 & 2 \\
\hline \multirow{2}{*}{ Ref-Reg. } & Mean & 22.39 & 38.67 \\
& Std & 27.98 & 43.801 \\
\hline \multirow{2}{*}{ Segm-Reg. } & Mean & 15.66 & 20.25 \\
& Std & 13.17 & 13.32 \\
\hline
\end{tabular}

An expert located 90 landmarks uniformly spread in the reference and moving mosaics. The landmarks were placed in the centroids of well-defined anatomical structures such as vessels and bronchioles perpendicular to the sectioning plane. The registration error was measured as the mean Euclidean distance between the landmark positions. The mean registration error for the proposed method was $41.19 \%$ smaller in average than for the reference method and corresponds to $1-2$ cell diameters (see Table 3).

\section{CONCLUDING REMARKS}

In this manuscript, we have presented a segmentation-based non-rigid registration method applied to the fusion of multiple stained histological sections. The method compares favorably with a state-of-the-art intensity-based non-rigid registration algorithm.

The poorer performance of the reference method is likely due to a relatively high similarity between the anatomical fea- tures in the histological images and their dissimilar appearance under different stains. Whereas the former limits the matching confidence, the latter negatively affects the convergence of intensity-based optimization algorithms.

The advantage of the proposed method is its robustness. Thanks to the multi-resolution approach, it captures and aligns the structures of interest at different scales. Furthermore, the merging and matching of the labels ensures that the segmentation of the reference and moving images represent indeed the same type of structures. An effort has been done to computationally optimize our method. Specifically, our superpixel approximation decreases the dimensionality of the subsequent segmentation and the simple similarity metric used contributes to the efficiency of the registration optimization.

\section{REFERENCES}

[1] G.J. Metzger, S. Dankbar, and et al., "Development of multigene expression signature maps at the protein level from digitized immunohistochemistry slides," PloS ONE, vol. 7, no. 3, pp. e33520, 2012.

[2] I. Arganda-Carreras, C. Sorzano, R. Marabini, and et al., "Consistent and elastic registration of histological sections using vector-spline regularization," 2006, vol. 4241, pp. 85-95.

[3] H. Bay, T. Tuytelaars, and L. Van Gool, "SURF: Speeded up robust features," in Euro Conf Comp Vision (ECCV'06). 2006, pp. 404-417, Springer.

[4] R. Achanta and el al., "SLIC superpixels compared to state-of-the-art superpixel methods," IEEE Trans Pat Anal Mach Intell, vol. 34, no. 11, pp. 2274-2281, 2012.

[5] A. Lucchi, K. Smith, R. Achanta, and V. Lepetit, “A fully automated approach to segmentation of irregularly shaped cellular structures in EM images," in Med Image Comp and Comp Assisted Inter, 2010, pp. 463-471.

[6] H.W. Kuhn, "The Hungarian method for the assignment problem," Naval Research Logistics Quarterly, vol. 2, no. 1-2, pp. 83-97, 2006. 